replacement therapy was complete since normal litters were sired, and the testes appeared normal on histological examination.

The atrophy of the thyroid and adrenal glands after removal of the pituitary was not repaired by injection of gonad stimulating hormone, but extracts containing the growth hormone maintained or restored the weight of these organs, although histologically the normal structure was not completely regained. Evans's results do not show whether it is the growth hormone or some other active principles in the extracts which are responsible for these effects. The cachexia commonly observed in hypophysectomised rats was also relieved by injections of the growth hormone.

The data on which the workers in the University of California base the conclusions briefly reviewed above are available in detail in the monograph now under notice. The methods described should be of value to other investigators and their results should form the basis of further research in this important field.

\title{
The British Association Tables of Bessel Functions
}

IN 1888 a Committee was appointed by the 1 British Association for the purpose "of considering the possibility of calculating tables of certain mathematical functions, and, if necessary, of taking steps to carry out the calculations, and to publish the results in an accessible form". The Committee had the late Lord Rayleigh for chairman, and 'Mr.' A. Lodge for secretary, and the other members were 'Sir W. Thomson', Cayley, Price, Glaisher, Greenhill and Hicks. Bessel functions were among the functions considered, and their calculation became the chief work of the Committee. Tables appeared in the Reports of the Association for 1889, 1893 and 1896. In 1907 the Committee reported on "The further tabulation of Bessel functions", and in 1909 stated that it was "also considering the advisability of collecting all existing tables of Bessel functions and publishing them in a form easily accessible to all students". During the next few years tables of Bessel functions of various types appeared regularly, and in $\mathbf{1 9 1 5}$ it was reported that "the order of calculation is being arranged in accordance with the real urgency of the tables, and the stage is now coming in sight at which the Committee will be able, as authorised already by the Association, to publish a volume of fairly complete tables of the more important transcendental functions".

The Committee's hopeful plans, like others, were, however, hurled to emptiness, and although the Committee remained in existence and many tables were printed in its reports, it was not until after the Glasgow meeting of the Association in 1928 that the preparation of the long foreseen volume was taken seriously in hand; and when in 1931 a volume appeared, it contained no tables of Bessel functions. The reasons for this, and the problems confronting the Committee, were explained in the preface :

". . . It was apparent from the first that the simple plan of reprinting existing material would produce a volume neither useful nor creditable. There were gaps in the ranges of the arguments of some of the functions, natural when the tabulation had been performed at different times for special purposes, but intolerable if tables were to be issued for general use. In the case of the Bessel functions, the functions tabulated did not form in any sense a complete collection. Lastly, the original tables offered no facilities for interpolation. Two years ago the Committee decided that these difficulties must not impede publication indefinitely, and that, if the Bessel functions were reserved for an independent volume, definite progress could be made."

Since 1929, therefore, the work on Bessel functions has been independent both of the pre. paration of volumes of tables of other functions and of the computation from time to time of special tables in response to current demands. The aim of the Committee is to publish as complete and uniform a set of tables of Bessel functions as possible; the majority will be derived from the reports, but several will be new. It is estimated that the tables will extend to nearly five hundred pages, and it is proposed to issue two volumes, partly to avoid an unwieldy book and partly to expedite publication. The material for the first volume is in an advanced state, more than threequarters of the estimated 280 pages being ready for the printer.

Unfortunately, financial difficulties have now to be overcome. It is not to be expected that an undertaking of this sort can be a paying proposition. The mathematicians concerned have given their services, but the mere cost of production will be between $30 s$. and $£ 2$ a page. The calls on the British Association are far more miscellaneous, far more extensive, than in the spacious days when $£ 500$ could cheerfully be voted for a single object. At the moment, a sum of $£ 150$, which includes a grant of $£ 50$ from the Royal Society for this purpose, has been set aside by the Council of the Association, but this is little more than an earnest of belief in the Committee's plan. Unless further help is forthcoming, there is a serious danger that the enterprise to which a multitude of volunteers have given their leisure during nearly half a century will be completed by the enthusiastic drudgery of the Committee which has accepted it as a heritage, only to rest-a manuscript almost too precious to be consulted-in a fire-proof safe. It ought to be sufficient, by directing attention to this possibility, to ensure that funds will be provided to enable the two volumes to be published and thus to make available the results of so many years of voluntary work on behalf of mathematical students and others. 